\title{
BASAL STRESS CONCENTRATIONS DUE TO ABRUPT CHANGES IN BOUNDARY CONDITIONS: A CAUSE FOR HIGH TILL CONCENTRATION AT THE BOTTOM OF A GLACIER
}

\author{
by \\ Kolumban Hutter \\ (Versuchsanstalt für Wasserbau, Hydrologie und Glaziologie, ETH-Zentrum, CH-8092 Zürich, \\ Switzerland) \\ and Vincent O.S. Olunloyo \\ (Department of Mechanical Engineering, University of Ilorin, llorin, Nigeria)
}

\section{ABSTRACT}

The existence of cold patches at the base of a glacier suggests that the sliding law will depend on these patches, which will essentially affect the viscosity constant. In a polythermal glacier, such as a glacier which is cold in its lower part and temperate in its upper part, basal boundary conditions change from no-slip to viscous sliding. It is anticipated that the viscosity constant of this sliding law will depend on the distance from the transition line between cold and temperate ice.

The mixed boundary conditions, namely noslip where the ice is cold and viscous sliding where it is temperate, induce large stresses and velocity changes close to the transition line. In fact, it is shown that, for a Newtonian fluid and all investigated discontinuities of boundary data, square-root singularities of the stresses will develop at the transition line. Asymptotic expressions for the basal stresses are derived. The explicit forms of these asymptotic expansions depend on the form of the spatial dependence of the sliding 1 aw and, furthermore, on the numerical values of the viscosity coefficient. It is, moreover, argued that the stress concentrations are sufficiently pronounced to account for the removal of basal rock especially in regions of high cleavage concentrations, the details again depending upon the sliding coefficients.

No mathematical details of the problen solved are presented as attention is focused on the physical processes.

\section{1) INTRODUCTION}

In seeking quantitative descriptions of glacier flow, attention is generally limited to glaciers which are either cold or temperate. With the exception of Fowler (1979|b]) and Hutter and 0lunloyo (1980) there are no investigations known to us which deal with the state of stress and velocity in a polythermal environment. Such glaciers are partly temperate and partly cold, and, depending on which of these states applies at the base, the ice is thought to be either fully adhering or sliding over its bed. It does not seem to be necessary to defend the postulate of the existence of polythermal glaciers, as there is sufficient field evidence corroborating cold ice in glaciers that are be1 ieved to be temperate at their beds. Harrison (1972) reports that water in some bore holes slowly freezes; Theakstone (1967) mentions that air temperatures in a tunnel beneath $50 \mathrm{~m}$ of ice near the margin of $\emptyset$ sterdalsisen in Norway remained almost constant at $-0.1^{\circ} \mathrm{C}$, and Vivian and Bocquet (1973) state that the ice roof of natural subglacial caverns in the Glacier d'Argentiere was often below the melting point. Robin (1976) has offered a "heat pump" argument to explain the existence of. cold patches at the base of a temperate glacier. It appears that spatial, as well as temporal, variations of basal pressure cause freezing of ice to rock.

Indirect evidence in support of this is the intermittent stick-slip motion of temperate glaciers reported by both Theakstone (1967) and Vivian and Bocquet (1973).

The cold patches at the base, described by Robin (1976), are assumed to be of the extent of the roughness amplitudes of the bed, or smaller, and must therefore affect the viscous sliding law supposed to apply at the smoothed-out surface of the global flow problem; in a first approximation, one may assume that they only affect the constant $C$ in the sliding law

$$
u_{\mathrm{b}}=C \tau^{m} \text {, }
$$

where $u_{b}$ is the basal sliding velocity, $\tau$ the corresponding shear traction, and $C$ and $m$ are constants, $m$ having the value $(n+1)$ (L.1iboutry 1979, weertman 1979) or $n$ (Fowler 1979[a]), $n$ being the exponent in Glen's flow law. $A$ proper treatment would have to take into account possible stress concentrations at the separation lines of cold and temperate ice and would have to include the possibility of tearing off some rocks from below. A mathematical solution to this problem does not seem to be in sight as it would consist of a complex, mixed boundaryvalue problem.

However, when the cold and temperate portions of the base extend over several thicknesses of the glacier, the stress-concentration problem where no-slip goes over into viscous sliding becomes mathematically amenable to analysis, at least approximately. This situation corresponds to a glacier which is cold in the 
lower and temperate in the upper parts. Our situation corresponcs to that discussed by Muller (1976). The reverse also occurs, and is believed to arise more frequently; it can be obtained from our analysis by simply selecting the angle $y$ as negative (this angle is described in section 2 and Figure 1). The abrupt change in boundary condition from no-slip to viscous sliding will induce large stress and velocity variations. The maximal stresses are to be expected at the "discontinuity" of boundary data.

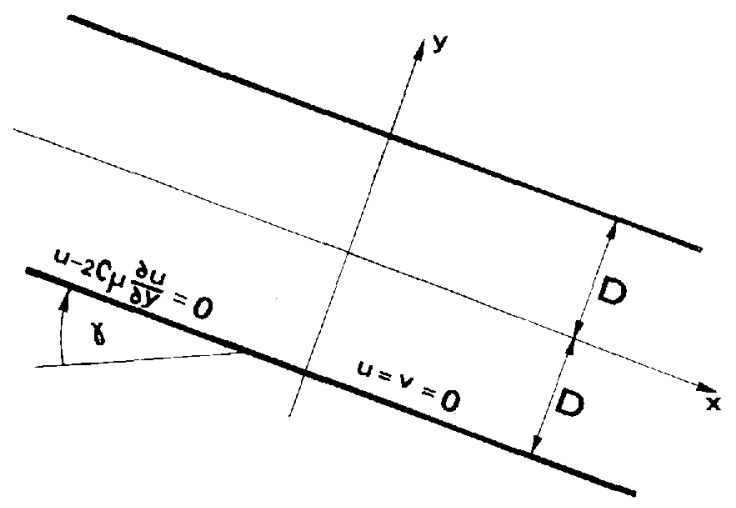

Fig. 1. Flow of ice down a strictly parallelsided slab can be modelled approximately by treating a channel-flow problem of ice held between two plates which are twice as far apart as the ice strip is deep. Inclination of the slope is $\gamma$ and the basal boundary conditions are no-slip $(u=v=0)$ and viscous sliding, respectively.

One question of geomorphological interest is whether these stresses are sufficiently high to tear off portions of the rock base. If they are, a partial explanation of the substantial amount of till observed at the bottom of an ice bore hole (Engelhardt and others 1978) could be explained. This, then, is the question to which we shall address ourselves, and indeed we shall demonstrate that the removal of rock from the base in the above-mentioned conditions is possible.

\section{2) MATHEMATICAL PROBLEM}

An infinite, parallel-sided ice slab under plane motion is studied, the upper half of the base of which adheres to the bed while the lower half of the bed is subject to viscous sliding. of interest is the stress distribution induced by gravity and, in particular, its behaviour near the abrupt change of boundary data. The exact problem based on non-linear rheology (e.g. Glen's flow law) is hardly amenable to an analytical solution, but by restricting attention to Newtonian behaviour approximate solutions can be obtained with reasonable effort. Although the Newtonian model suffers the disadvantage of not being materially realistic for ice, it is a useful one because it allows the solution of a wel1-defined mathematical problem free of $a d$ hoc assumptions. The same applies to the many other linear models introduced into the ice literature (Nye 1969 and 1970, Kamb 1970, L7iboutry 1975, Morland 1976 Ta] and $1976\left[\mathrm{~b}^{-}\right]$, Morris 1979, Hutter and 0lunloyo 1980). Specifically, consider slow flow of a Newtonian viscous fluid held between two parallel planes whose inclination angle is $\gamma$ and which are at a distance $2 D$ apart (Fig. 1). Let $(x, y)$ be a Cartesian coordinate system; $x$ is midway between the two planes and $y$ perpendicular to it, so that the two planes confine the fluid to the region $|y| \leq D$. We assume mixed boundary conditions, namely no-slip for $x>0$ and viscous sliding for $x \leq 0$. The sliding law will be taken in the form of Equation(1) with $m=1$ and a coefficient $C(x)$ that varies with $x$. This $x$-dependence will be further elaborated below. Let $\rho$ be the density, $g$ the gravity constant, $\mu$ the dynamic viscosity, $(u, v)$ the longitudinal and transversal velocity components, and $p$ the pressure. The governing equations describing steady flow through the above channel are given by the differential equations (Fig.1):

$$
\begin{aligned}
& \frac{\partial u}{\partial x}+\frac{\partial v}{\partial y}=0, \\
& \frac{\partial p}{\partial x}=\rho g \sin \gamma+\mu \nabla^{2} u, \\
& \frac{\partial p}{\partial y}=-\rho g \cos \gamma+\mu \nabla^{2} v,
\end{aligned}
$$

subject to boundary conditions:

$$
\begin{array}{ll}
u=v=0, & |y|=D, x>0, \\
u-c_{u} \frac{\partial u}{\partial y}=v=0, & |y|=D, x \leq 0 .
\end{array}
$$

Equations (2) express mass balance for an incompressible body and momentum balance when convective acceleration terms are neglected. The first of Equations (3), on the other hand, expresses the no-slipboundary condition, whereas the second of (3) is the viscous sliding law. Because of its physical significance the latter warrants further attention.

We assume the sliding coefficient to vary along the temperate part of the boundary, typically as shown in Figure 2. At large values

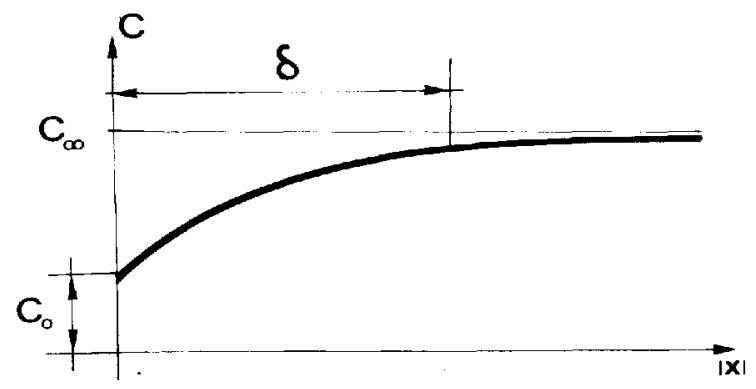

Fig. 2. A typical assumed variation of the viscosity coefficient in the basal sliding law. At the transition point where temperate ice becomes cold $C=C_{0}$ is minimal and may even have the value $C_{0}=0$. This would then correspond to a continuous transition from no-slip to viscous sliding.

of $|x|$, effectively for $|x|>\delta$, where $\delta$ is a tronsition length, the sliding coefficient is nearly constant with a value approaching $C$ as $|x| \rightarrow \infty$. For $0 \leq|x| \leq \delta, C$ depends on $x$, starting at $x=0^{-}$with a value $C_{0}$ and then monotonically increasing, approaching the value $C_{\infty}$ effectively at $x=-\delta$. We regard this variation as a reasonable model for the description of the transition from viscous sliding to no-slip. Close to $x=0$ a decrease of $C$ with decreasing $|x|$ is most likely to occur since the number of cold patches will increase as the cold portion of the base is approached. We assume that the transition depth $\delta$ must be small compared with the glacier thickness $D$; it is, however, physically not clear whether $C_{0}$ differs from zero or must vanish. Fowler's work (1979[a]) 
suggests that $C_{0}$ should vanish, and he claims that in this case both stresses and velocities remain bounded at the point of discontinuity of boundary data. Hutter and 0lunloyo (1980), on the other hand, have shown that for $C_{0}=C_{\infty} \rightarrow \infty$ (corresponding to perfect slip), the stresses show half-order singularities at the point $\approx=0$. It is thus expected that the details of the stress and velocity distributions will depend on the transition properties of the sliding law in the neighbourhood of the transition point "temperate-cold". As a consequence, the failure criterion for the rock beneath will also depend on the pertinent details of the sliding law. Mathematically, the boundary value problem set forth by Equations (2) and (3) can be shown to be reducible to a problem of the Wiener-Hopf type (Hutter and 0lunloyo 1980), and it is in the form of the latter that an approximate solution can be found. Symmetry of the mathematical formulation about $y=0$ is obtained by having identical boundary conditions on $y= \pm D$, by redefining pressure to incorporate transverse components of the gravity forces, and by imposing zero transverse velocity and zero shear traction, but not zero normal traction, on the surface $y=0$. This is not entirely realistic as shear and normal tractions should vanish on the true free surface, but, for perfect slip on the temperate side, calculations indicate that $p$ on $y=0$ is small and does not affect the singularity. The details of the construction of the solution of the Wiener-Hopf problem are very elaborate (as shown by 0lunloyo and Hutter, unpublished). In the following section the most important results pertinent to the problem at hand will be discussed.

\section{3) BASAL STRESSES NEAR THE POINT OF ABRUPT}

CHANGES OF BOUNDARY DATA

$A$ possible mathematical representation for the dimensionless viscosity coefficient in the sliding law as depicted in Figure 2 is

$$
C(x)= \begin{cases}C_{\infty}-\left(C_{\infty}-C_{0}\right) \exp (-|\bar{x}| / \delta), & x<0, \\ 0 & , x \geq 0 .\end{cases}
$$

In this equation the various forms of $C$ are inade dimensionless with $D /(2 \mu)$ so that $C_{\text {dim }}=C D /(2 \mu)$ Similarly, $\bar{x}$ and $\delta$ are dimensionless lengths based on $D$, the glacier thickness. With $c_{\text {dim }}=u_{\mathrm{b}} / \tau_{\mathrm{b}}$ and with $u_{\mathrm{b}}=100 \mathrm{~m} \mathrm{a}-1, \tau_{\mathrm{b}}=10^{5} \mathrm{~Pa}$,

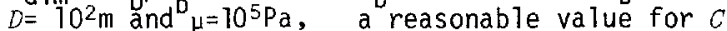
is about 2 .

The Wiener-Hopf problem based on Equations (2), (3) and (4) was solved by approximating the kernel of the Wiener-Hopf equation. Two approximations have been found. The first is valid, provided that $C=C \rightarrow \infty$ and when the boundary condition at the temperate side is that of perfect slip (Hutter and 0lunloyo 1980). The other applies for viscous sliding according to the functional representation of Equation (4) and holds for any finite value of $C_{\infty}$ and any value of $C_{0} / C_{\infty}$ in the interval $[0,1]$.

Let $\Sigma_{x}, \Sigma_{y}, \theta$ be dimensionless normal and shear stresses at the base, respectively, which have no dimensions with respect to the basal shear stress $p g D$ sin $y$ of the strictly parallel-sided slab. Hence, orders of magnitudes to be expected for $\Sigma_{x}, \Sigma_{y}$ and $\theta$ in a strictly parallel-sided slab are 10 and 1, respectively. For the special case that $C_{0}=C_{\infty} \rightarrow \infty$ the functions $\Sigma_{x}(x), \Sigma_{y}(x)$, and $\theta(x)$ have been evaluated. For the more general relationship of Equation (4) explicit formulas for the basal stresses are probably difficult to find, but asymptotic re- presentations for small $|x|$ can be found with reasonable effort. The expressions have the general form

$$
f(\bar{x}, 1)= \begin{cases}\sum_{v=-1}^{\infty} f_{v / 2}^{f^{\prime}} \bar{x}^{v / 2}, & \text { as } \bar{x} \rightarrow 0^{+}, \\ \infty \\ \sum_{v=-1} b_{v / 2}^{f} \bar{x}^{v / 2}, & \text { as } \bar{x} \rightarrow 0^{-},\end{cases}
$$

in which $f$ stands for either shear stress, overburden, or longitudinal stress $\theta, \Sigma_{y}$, and $\Sigma_{x}$ Notice that $u$ may have the value -1 , so that the stresses may have a square-root singularity. Indeed, calculations show that the shear stress is regular as $x=0^{-}$is approached from the temperate side, but becomes singular when $x=0^{+}$ is approached from the cold side. The singularity is of square-root type and can be integrated. When integrated to lowest order one obtains

$$
\theta(\bar{x}, 1)= \begin{cases}a_{0}{ }^{\theta} & , \text { as } \bar{x} \rightarrow 0^{+}, \\ b_{-\frac{1}{2}}^{\ominus}|\bar{x}|^{-\frac{1}{2}} & , \text { as } \bar{x} \rightarrow 0^{-},\end{cases}
$$

with coefficients which are functions of $C_{0}, C_{\infty}$, and $\delta$. Detailed analysis shows that $\delta \Theta^{\Theta}$

is finite and differs from zero for al $1^{\frac{1}{2}}$ values of $C_{0} / C_{\infty}$ in the interval $[0,1]$. Consequently the square-root singularity persists even in case the coefficient function $C(x)$ is continuous at $x=0$. Explicit calculations are too elaborate for arbitrary values of the transition length $\delta$, but become much simplified when $\delta=\pi^{-1}$. This value corresponds to a transition length which is about one-third of the glacier thickness. This should be borne in mind. The basal shear stress reads

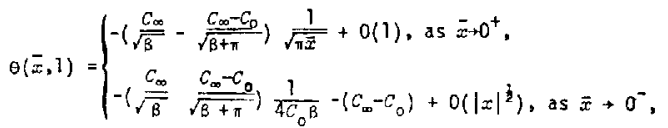

where $B$ is a function of $C_{0}$ and $C_{\infty}$ and can be read off from Table I. This table shows the product $C_{\infty} \beta$ as a function of $\left(C_{0}-C_{\infty}\right)$; see also Figure 3. It should also be noticed that in the limit $C_{0}=C_{\infty} \rightarrow \infty$ Equation (7) breaks down and results must be obtained from the earlier investigation (Hutter and 01unloyo 1980).

\begin{tabular}{|c|c|c|}
\hline $1-\frac{C_{0}}{C_{\infty}}$ & B & Residue \\
\hline 0.0 & 3.1811 & 0.0002 \\
\hline 0.1 & 3.1793 & -0.0003 \\
\hline 0.2 & 3.1777 & 0.0002 \\
\hline 0.3 & $3.176 \quad 0$ & -0.0007 \\
\hline 0.4 & 3.1746 & 0.0000 \\
\hline 0.5 & 3.1733 & -0.0003 \\
\hline 0.6 & 3.1720 & 0.0003 \\
\hline 0.7 & 3.1707 & -0.0004 \\
\hline 0.8 & 3.1696 & 0.0000 \\
\hline 0.9 & 3.1685 & -0.0002 \\
\hline 1.0 & 3.1675 & 0.0000 \\
\hline
\end{tabular}

TABLE I. COEFFICIENT $B$ AS A FUNCTION OF THE PARAMETER ( $\left.1-\frac{C_{0}}{C_{\infty}}\right)$ AND RESIDUE

INDICATING THE ACCURACY OF THE ROOT 


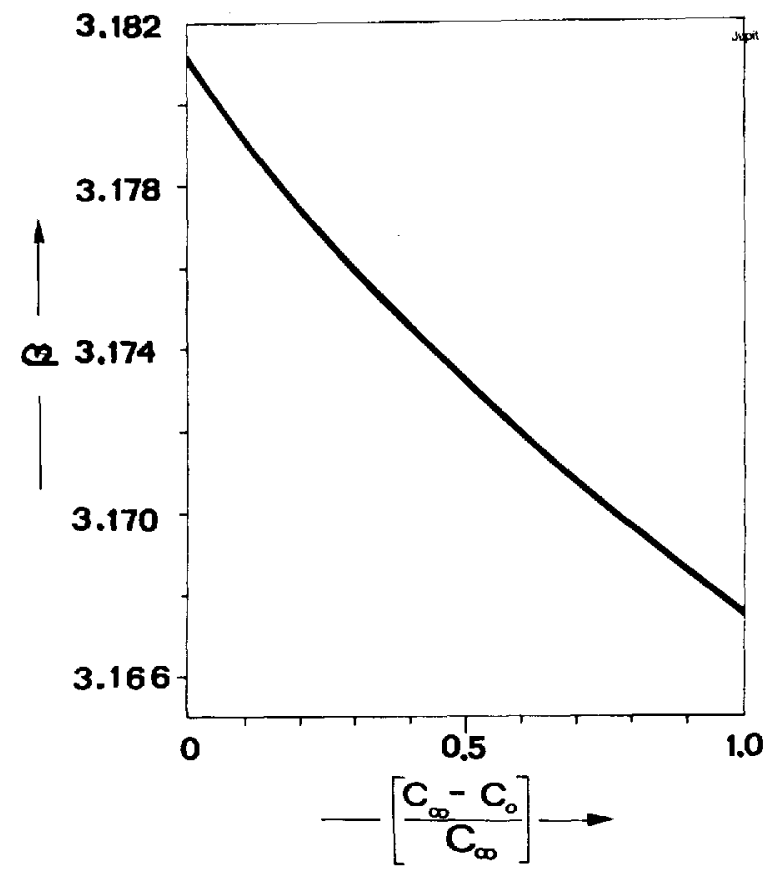

Fig. 3. Functional dependence of the coefficient $B$ as a function of $\left(1-\frac{C_{O}}{C_{\infty}}\right)$.

For the longitudinal normal stress and the overburden pressure, the singular behaviour is more sensitive to variations in boundary data than is the case for the shear tractions. When the perfect slip condition is applied at the temperate side, both stress components $\Sigma_{x}$ and $\Sigma_{y}$ are singular at $x=0$ with square-root singularities. On the other hand, when the viscous sliding law (1) is used, the singularity in the longitudinal stress $\Sigma_{x}$ persists while overburden pressure is now finite. This is an indication that with a decrease in singuiarity in boundary data smoother stress fields should be obtained. To be sure, asymptotic representations for $\Sigma_{x}$ and $\Sigma_{y}$ are

$$
\Sigma_{x}(\bar{x}, y)=\cot \varphi+ \begin{cases}B+0\left(\vec{x}^{2}\right) & \text { as } \bar{x}+0^{+}, \\ -2\left(\frac{C_{\infty}}{\sqrt{\bar{B}}}-\frac{C_{\infty}-C_{O}}{\sqrt{\bar{\beta}+\pi}}\right) \frac{1}{\sqrt{\pi \mid \bar{x} T}-\frac{\bar{x}}{\bar{C}_{0}},} \text { as } \bar{x}+0^{-},\end{cases}
$$

and

$$
\varepsilon_{y}(\bar{x}, 1)=\cot Y+\left\{\begin{array}{l}
B+O\left(\bar{x}^{2}\right), \text { as } \quad \bar{x}+0^{+}, \\
-\frac{\bar{x}}{\bar{C}_{0}}+O\left(\bar{x}^{2}\right), \text { as } \bar{x} \rightarrow 0^{-}
\end{array}\right.
$$

with

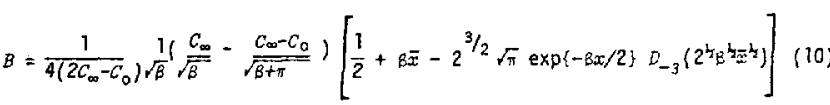

where $D_{-3}($.$) is the parabolic cylinder function.$ of particular interest is the coefficient of the $x^{-1 / 2}$ term in Equations (7) and ( 8 ) because it determines the dominant parts of the stresses $\theta$ and $\Sigma_{x}$. Values for this coefficient are plotted as shown in Figure 4. This graph is for the coefficient of $\Sigma_{x}$ which is twice as large as the corresponding coefficient for 0 . The singular behaviour expressed by square-root singularities occurring in Equations (7) and (8) points at a possibility of failure or removal of basal rock; in fact, square-root singularities are also expected in the principal stresses at the base so that from a theoretical point of view both ice

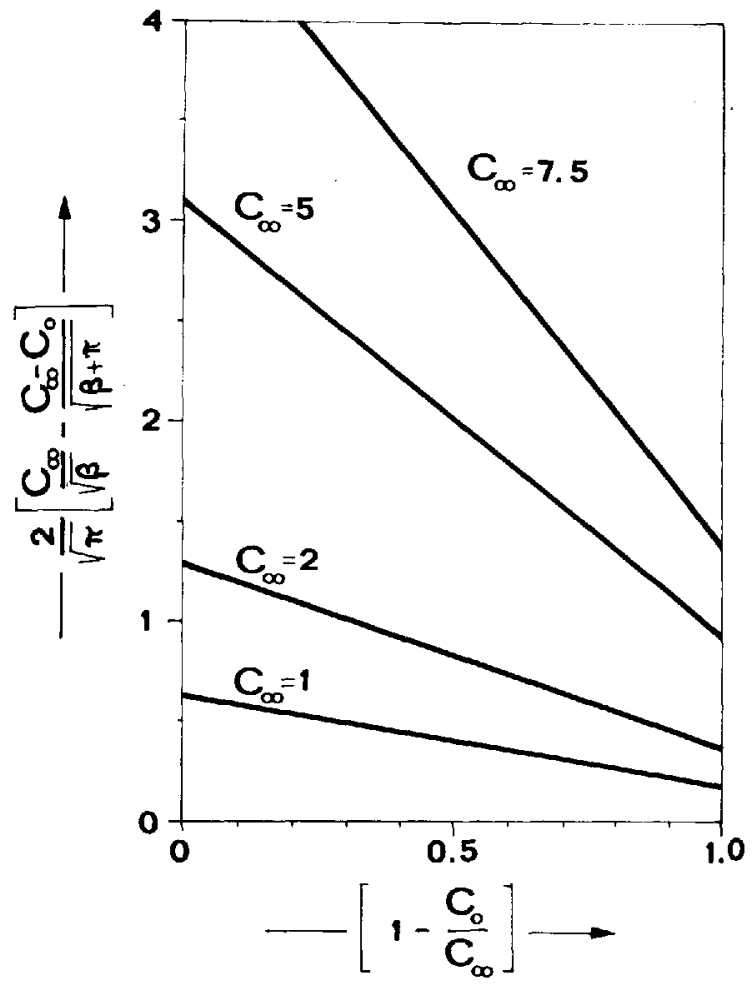

Fig. 4. Plot of the coefficient of the singular term in the stress expansion as given in the Equations (7) and (8).

and rock must fail if a failure criterion under tensile stress is assumed. Asymptotic expressions for the principal stresses $\Sigma_{1}$ and $\Sigma_{2}$ can be obtained by using the formula

$\Sigma_{1,2}=\frac{\Sigma_{x}+\Sigma_{y}}{2} \pm\left[\left(\frac{\Sigma_{x}-\Sigma_{y}}{2}\right)^{2}+\tau^{2}\right]^{\frac{1}{2}}$

and upon substitution of the foregoing expansions. The dominant term is

$\Sigma_{1,2} \cong 2 z_{-\frac{1}{2}}^{\Theta}|\bar{x}|^{-\frac{1}{2}}$, as $\bar{x} \rightarrow 0^{ \pm}$.

It is irteresting to observe that the principal stresses are singular on both sides of the discontinuity of boundary data. In contrast to the stresses, forces remain finite, however, because it is possible to integrate the singularities. Therefore integrating the stresses over a small length $E$ yields (in physical dimensions):

$\vec{r}_{1}=2 \cdot 2.0 \mathrm{~g}|\sin \gamma| D b_{-\frac{1}{2}}^{\Theta}\left(\frac{\varepsilon}{D}\right)^{\frac{1}{2}}$

as a typical value for the tensile force acting perpendicularly to a possible failure plane. This tensile force arises for both positive and negative slope $\gamma$ but acts on planes of different orientations in these instances.

A typical value for $b \theta$, is about 1 or 2 so that with $p=9 \times 10^{2} \mathrm{~kg} \mathrm{~m}^{-3},{ }^{-\frac{1}{2}} \mathrm{~g}=9.8 \mathrm{~m} \mathrm{~s}^{-2}$, siny $=$ $10^{-2}, D=10^{2} \mathrm{~m}$ and $\varepsilon=10^{-1} \mathrm{~m}, E=2 \times 10^{3} \mathrm{~N}$. Hence the average maximal tensile stress acting on an element of $10^{-2} \mathrm{~m}^{2}$ area in a possible failure plane is about $\sigma_{1}=2 \times 10^{5} \mathrm{~Pa}$. This is in the order of the failure strength of ice under uniaxial tension and is probably also sufficiently high for rocks, provided they contain a sufficient amount of microcracks. Of course, on a smaller scale, mean stresses are larger. 
4. SOME FINAL REMARKS

The purpose of the above linear analysis is twofold: first, it can be used to substantiate the supposition that stress concentrations at the melting point of the base in a polythermal glacier are sufficiently high to tear off from the base rock pieces as seen in field observations; second, the analysis gives information about the nature of this stress concentration, as it depends on the variation of the sliding coefficient $C$. We reported that for the sliding law obeying Equation (4) stress singularities persist even when $C(x)$ is continuous (but not differentiable) at $x=0$. This does not imply that stresses which are large but bounded at $x=0$ cannot exist, but that bounded stress would require a smoother transition of the sliding coefficient from cold to temperate sides. Morland (private communication) suggests such a smoother transition by requiring a viscous sliding condition to be valid everywhere at the boundary, whereby the sliding coefficient $c$ would approach infinity as the temperate side is approached. We have not analysed this case, and the problem is a global one, "local" stresses in the basal layer are likely to be concentrated, and induce bed failure. This points clearly at the difficulty associated with the sliding condition. Nothing is known at present about the sliding law close to the coldtemperate transition zone and the associated stress concentrations.

Removal of rock from the base can also be explained by mechanisms other than stress concentrations due to abrupt changes in boundary data only. Röthlisberger and Iken (1981) give such an alternative based on a regelation argument when basal cavities are formed. Their model explains how pieces of basal rock, that have already broken loose, are transported by the ice; our model suggests why the basal rock fractures into pieces.

Finally it should be mentioned that our calculations can only be regarded as approximations because effects of regelation and nonlinearities of the creep behaviour have been omitted. It is unlikely that these effects can be included in a quantitative analysis. Yet we think that our model gives orders of magnitude correctly.

\section{REFERENCES}

Engelhardt H F, Harrison W D, Kamb W B 1978 Basal sliding and conditions at the glacier bed as revealed by bore-hole photography. Journal of Glaciology 20(84): 469-508

Fowler A C 1979[a] A mathematical approach to the theory of glacier sliding. Jourmal of Glaciology 23(89): 131-141

Fowler A C 1979[b] The use of a rational model in the mathematical analysis of a polythermal glacier. Joumaz of Glaciology $24(90): 443-456$

Harrison W D 1972 Temperature of a temperate glacier. Jourmal of Glaciology 11(61): 15-29

Hutter K, Olunloyo $V$ OS 1980 on the distribution of stress and velocity in an ice strip, which is partly sliding over and partiy adhering to its bed, using a Newtonian viscous approximation. Proceedings of the Royal Society of London A 373 (1754): 385-403

Kamb W B 1970 sliding motion of glaciers: theory and observation. Reviews of Geophysics and Space Physics 8(4):673-728
L1iboutry L A 1975 Loi de glissement d'un glacier sans cavitation. Annazes de Geophysique $31(2)$ : 207-225

Lliboutry L A 1979 Local friction laws for glaciers: a critical review and new openings. Joumal of Glaciology 23(89): $67-96$

Morland L W 1976[a] Glacier sliding down an inclined wavy bed. Joumal of Glaciology $17(77): 447-462$

Morland L W 1976[b] Glacier sliding down an inclined wavy bed with friction. Joumal of Glaciology 17(77): 463-477

Morris E M 1979 The flow of ice, treated as a Newtonian viscous liquid, around a cylindrical obstacle near the bed of a glacier. Joumal of Glaciology 23(89): $117-129$

Müller $F 1976$ On the thermal regime of a highArctic valley glacier. Jourmal of Glaciozogy 16(74): 119-133

Nye $J F 1969$ A calculation on the sliding of ice over a wavy surface using a Newtonian viscous approximation. Proceedings of the Royal Society of London A 311(1506): 445467

Nye J F 1970 Glacier sliding without cavitation in a linear viscous approximation. Proceedings of the Royal Society of London A 315(1522): 381-403

Robin G de Q 1976 Is the basal ice of a temperate glacier at the pressure melting point? Joumal of Glaciology 16(74):183196

Röthlisberger $H$, Iken A 1981 Plucking as an effect of water-pressure variations at the glacier bed. Annals of Glaciology 2: 57-62

Theakstone W H 1967 Basal sliding and movement near the margin of the glacier osterdalsisen, Norway. Joumal of Glaciology 6(48): 805-816

Vivian R A, Bocquet G 1973 Subglacial cavitation phenomena under the Glacier d'Argentiere, Mont Blanc. Journal of Glaciology 12(66): 439-451

Weertman $J 1979$ The unsolved general glacier sliding problem. Journal of Glaciology 23(89): $97-115$ 